\title{
Tradición e innovación en la prehistoria andina de San Pedro de Atacama
}

AMY OAKLAND ${ }^{1}$

\section{RESUMEN}

El árido Desierto de Atacama ha permitido una preservación poco común en el mundo y por esa razón la posibilidad de estudiar una vasta cantidad de textiles prehistóricos y otros materiales perecederos. La mayoría de las reconstrucciones de la prehistoria de Atacama, basadas en criterios cerámicos identifican un solo grupo indígena habitando en los oasis de San Pedro. Puesto que las ofrendas de cerámica no siempre han estado presente en los enterramientos del Area Centro Sur Andina, no se puede esperar que estas ofrendas provean la misma definición íntima, a nivel de identificación personal, como lo provee el análisis de los textiles. A juzgar por los tejidos modernos de las comunidades indígenas del centro sur de los Andes, éstos son notables por sus colores brillantes e intrincados y por sus diseños específicos a cada identidad étnica. En relación a estas cuestiones de identidad, no se ha encontrado ningún registro arqueológico que sugiera que el tejido andino prehistórico haya sido muy diferente al presente. En este sentido los estilos múltiples de ropa representados en el cementerio Coyo Oriental describen enterramientos multiétnicos, reflejando posibles convivencias bi y/o multiétnicas entre la población del oasis de Coyo.

\section{ABSTRACT}

The arid Atacama Desert has allowed the uncommon preservation of a large quantity of prehistoric textiles excavated in the cemetery of Coyo Oriental. Although many reconstructions of Atacama prehistory discuss ceramic associations and identify a single indigenous population residing in the oases of San Pedro, the present analysis focuses on textile style and suggests that the multiple clothing styles represented in the ancient Coyo Oriental cemetery identifies multiethnic burials and possible multiethnic communities. The discussion focuses on three women whose separate textile styles identify them as belonging to larger groups who maintained a distinctive textile tradition possibly related to their original ethnic affiliations: one highland related to Tiwanaku, the other local with longer ties to the Chilean desert region.

1 California State University, Hayward, California, ESTADOS UNIDOS.

\section{Estilo y lugar}

En los años 1971 y 1975 , Le Paige $(1974,1975)$ excavó 216 tumbas que datan entre el siglo sexto y décimo, en el cementerio Coyo Oriental, localizado al este del ayllu de Coyo en San Pedro de Atacama. No todos los fardos funerarios se han preservado de igual forma. Sin embargo, las evidencias sugieren que los paquetes incluyen varias capas de túnicas, mantas y tocados, atados con lazos de fibra vegetal y de camélidos. Comúnmente, los fardos estaban rodeados por armas, restos de comida, bolsas, canastas y cerámica.

Algunos investigadores de la Subárea Circumpuneña han querarquizado la presencia sincrónica de diversas culturas en los oasis atacameños. Se sabe que ciertas investigaciones previas han planteado que el flujo Tiwanaku se correlaciona con la llegada de gentes concretas desde las cabeceras del altiplano, a modo de colonias, ocupando los oasis atacameños: "en plena convivencia con las autoridades locales, afianzados a través de alianzas y fuerte proselitismo religioso (Bittmann et al. 1978: 42). Por otra parte, Núñez (1992: 54) ha reiterado que el flujo Tiwanaku en estos oasis ocurrió a través de ocupaciones directas multiétnicas, recurrentes en estos oasis con suficiente permanencia "amparados por eventuales alianzas interétnicas".

Por lo menos dos grupos diferentes de hombres, mujeres y niños fueron enterrados en el cementerio Coyo Oriental y, sin duda, estos grupos compartieron el oasis de Coyo entre el siglo VI y X DC (Tabla 1). Uno de estos grupos era probablemente local, derivado de una tradición antigua de San Pedro, y el otro grupo posiblemente conectada con el altiplano boliviano y el centro cultural, político y económico de Tiwanaku (300-1000 DC). Sin duda, la preeminente influencia de Tiwanaku en el Area Centro Sur Andina fue paralela a la ocupación de Coyo.

Los artefactos de Tiwanaku en San Pedro de Atacama fueron originalmente descritos como 


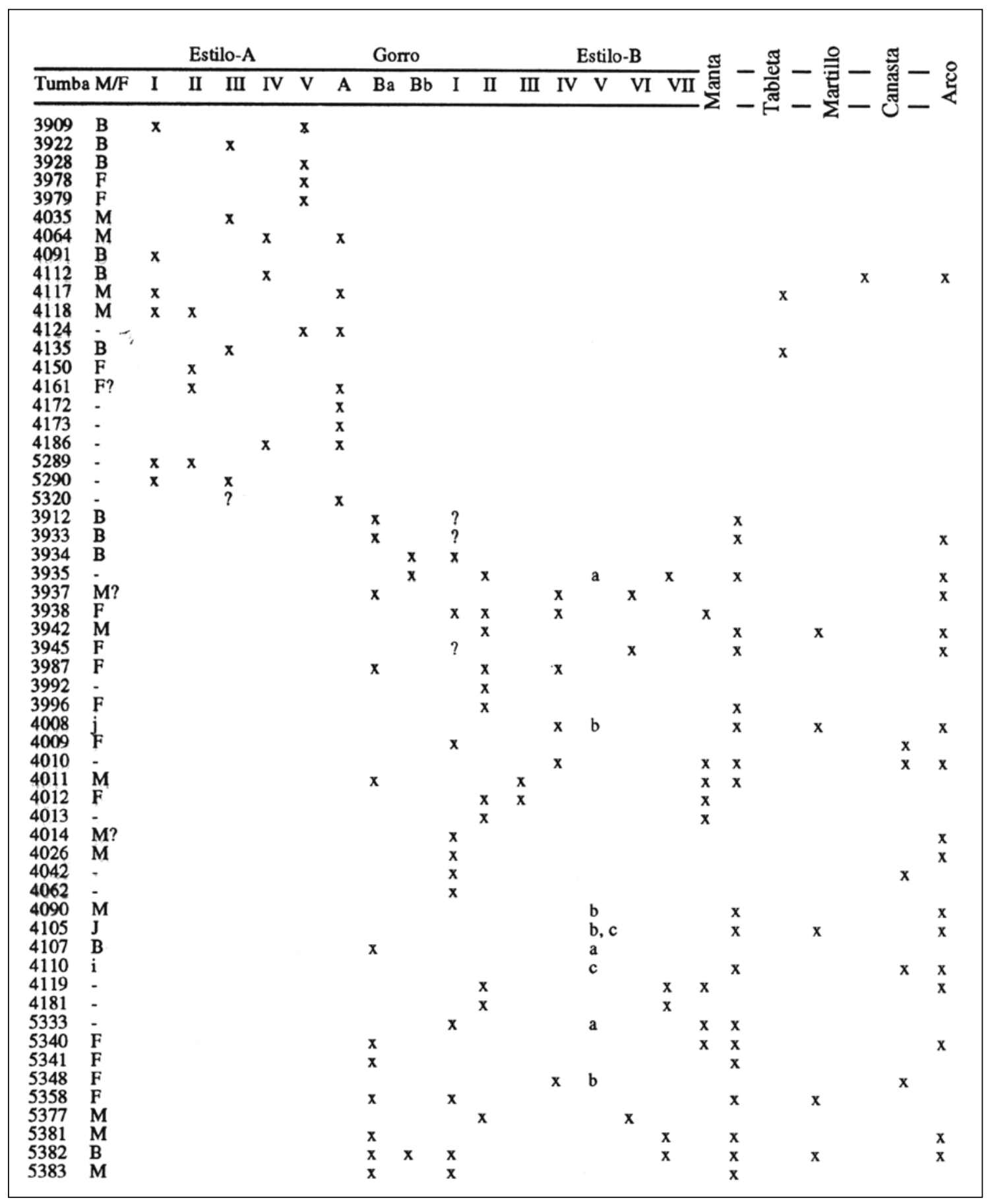

Tabla 1. Textiles y artefactos asociados con los estilos de los Grupos A y B de Coyo Oriental. 
artículos de intercambio provenientes del tráfico de llamas (Browman 1980), aunque el movimiento caravanero giratorio suma al trueque la ocupación directa de espacios extraaltiplánicos con traslado de bienes exóticos (Núñez y Dillehay 1979). En este sentido, propongo que los textiles y artefactos son objetos de importación, traídos directamente por poblaciones extranjeras. El presente análisis sostiene esta hipótesis. En efecto, túnicas y mantas tejidas en técnica de tapiz Tiwanaku descubiertas en Coyo, mencionadas en un artículo anterior (Oakland 1986), se conectan a un grupo específico de prendas de vestir hechas con una técnica simple (faz de urdimbre). Estos textiles son distintos de las prendas de vestir de estilo local, evidentes no sólo en Coyo, sino también en una gran variedad de oasis de otras localidades aledañas sugiriendo así una propagación más extensa del estilo local.

Ultimamente el concepto de estilo ha generado una gran riqueza en la discusión antropológica (Sackett 1982, 1990; Conkey y Hastorf 1990). Mi contribución a la literatura (Oakland 1992) se ha basado precisamente en el análisis de los textiles del cementerio Coyo Oriental, en donde se observa que el estilo específico de los textiles fue mantenido por los grupos étnicos prehistóricos como el emblema principal de su identificación a nivel de grupo, parecido a la forma en que lo hacen las comunidades andinas contemporáneas.

Para poder especificar y visualizar esta problemática se revisarán las ropas y los artefactos recuperados de la llamada "mujer de la manta roja Tiwanaku" enterrados en la tumba 4012 con una manta roja en tapicería, para contrastar el estilo textil con otra tumba llamada "dos hermanas locales" (una familia hipotética: dos mujeres compartiendo la misma tumba 3978-3979 con textiles muy parecidos). Los diferentes estilos fueron usados en Coyo simultáneamente, como lo sugieren las asociaciones cerámicas y determinaciones radiocarbónicas (600-900 DC) (Tabla 2). Originalmente, los grupos fueron identificados por varios criterios, especialmente el tocado masculino, el cual fue usado por varios grupos pero a nivel distintivo de cada uno de ellos. El centro del tocado es particularmente indicativo: los grupos locales usaron diseños espirales en técnica de anillado (looping) (Figura 1), mientras que los grupos del altiplano usaron diseños en forma de diamante, en técnica de anudado o knotting (Figura 2).

El nivel social es difícil de determinar entre los diferentes grupos. El entierro de la mujer del altiplano fue equipado con más textiles que el de las mujeres locales, pero es notable que su compañera enterrada en la misma tumba tuviera muy pocos textiles. Dentro de la colección completa el examen de los artefactos no textiles sugiere que un nivel más jerárquico fue posiblemente dado al grupo del altiplano en Coyo. Sin embargo, los textiles iluminan acerca de la distinción y experiencia técnica aparente en ambos grupos.

El estudio de la vida diaria de Coyo debe considerar aquellos componentes que han sobrevivido de los ritos mortuorios. Desafortunadamente, los textiles andinos prehistóricos preservados en sitios habitacionales son escasos. La información dada por los fragmentos de tela esparcidos en los pisos de viviendas prehistóricas contrastan obviamente con aquellos fragmentos encontrados en los cementerios. Puesto que los textiles de los muertos suelen demostrar evidencia de uso y reparación, es posible asumir que muchas de estas ropas representan el vestido diario de los habitantes locales, en este caso de Coyo.

\begin{tabular}{|c|c|c|c|c|c|}
\hline Coyo & $\mathbf{C}^{\mathbf{1 4}}$ & $\mathbf{C}^{\mathbf{1 3} / \mathbf{C}^{\mathbf{1 2}}}$ & Fecha absoluta & $\mathbf{m i ́ n} / \mathbf{m a ́ x}$ & Beta \\
\hline 5383 & $1330 \pm 60$ & $1430 \pm 60$ & $520 \mathrm{DC}$ & $435-680 \mathrm{DC}$ & 33858 \\
\hline 5341 & 1210160 & $1320 \pm 60$ & $630 \mathrm{DC}$ & $603-862 \mathrm{DC}$ & 33856 \\
\hline 4064 & $1180 \pm 80$ & $1310 \pm 80$ & $640 \mathrm{DC}$ & $580-890 \mathrm{DC}$ & 33855 \\
\hline 4012 & $1290 \pm 70$ & $1310 \pm 70$ & $640 \mathrm{DC}$ & $601-635 \mathrm{DC}$ & 33853 \\
\hline 5347 & $1040 \pm 180$ & $1155 \pm 80$ & $795 \mathrm{DC}$ & $670-1020 \mathrm{DC}$ & 33857 \\
\hline 4026 & $1010 \pm 170$ & $1100 \pm 70$ & $850 \mathrm{DC}$ & $770-1147 \mathrm{DC}$ & 33854 \\
\hline
\end{tabular}

Tabla 2. Fechas radiocarbónicas de seis tumbas de Coyo Oriental (Oakland 1992). 


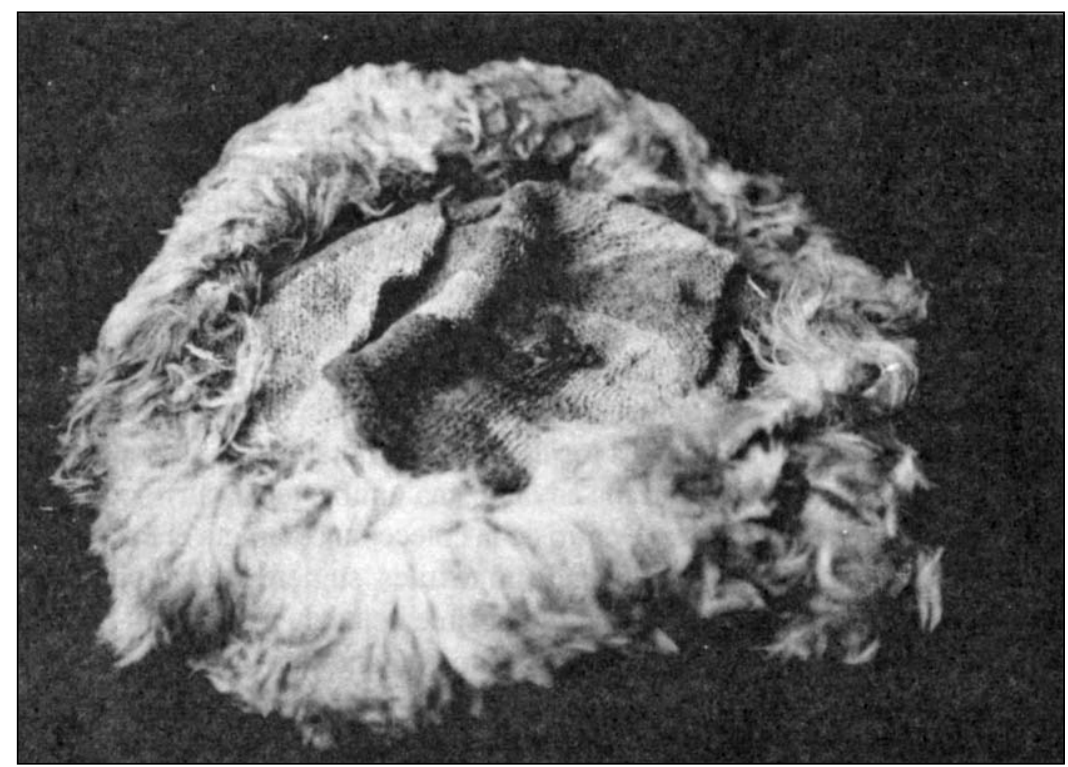

Figura 1. Un tocado asociado al estilo del Grupo A en Coyo (tumba 4064). Se advierte una trenza de fibra vegetal cubierta con tiras cortadas de piel de camélidos. El centro está tejido en técnica de anillado (looping).

\section{Mujer, sociedad y prehistoria}

El enfoque en este artículo sobre las mujeres es pertinente a la literatura antropológica (Gero y Conkey 1991) y de la historia del arte (Miller 1988) publicada recientemente en donde varios autores han creado una imagen más clara de la prehistoria. Estudios de etnografía contemporánea describen la sociedad andina como un sistema fluido, donde los papeles específicos de cada género son sobreentendidos como fijos, sin embargo, no siempre se han mantenido así. Allen (1988: 78) describe una situación común en la aldea de Songo, en el altiplano del sur peruano:

"En la división complementaria del trabajo [...] las mujeres hilan la lana, tejen en el piso en telares horizontales y cosen las ropas de la familia. Los hombres tejen sus propios sombreros, trenzan cuerdas finas de lana para arrear a los animales, tejen en un telar vertical con pedales y hacen un poco de carpintería simple."

Después de hablar de la versión aceptada de los roles complementarios, Allen (1988: 78) menciona que "los hombres hilan tanto como las mujeres y he visto hombres tejiendo finos ponchos, cinturones y bolsas para la coca con complejos diseños en telares horizontales."

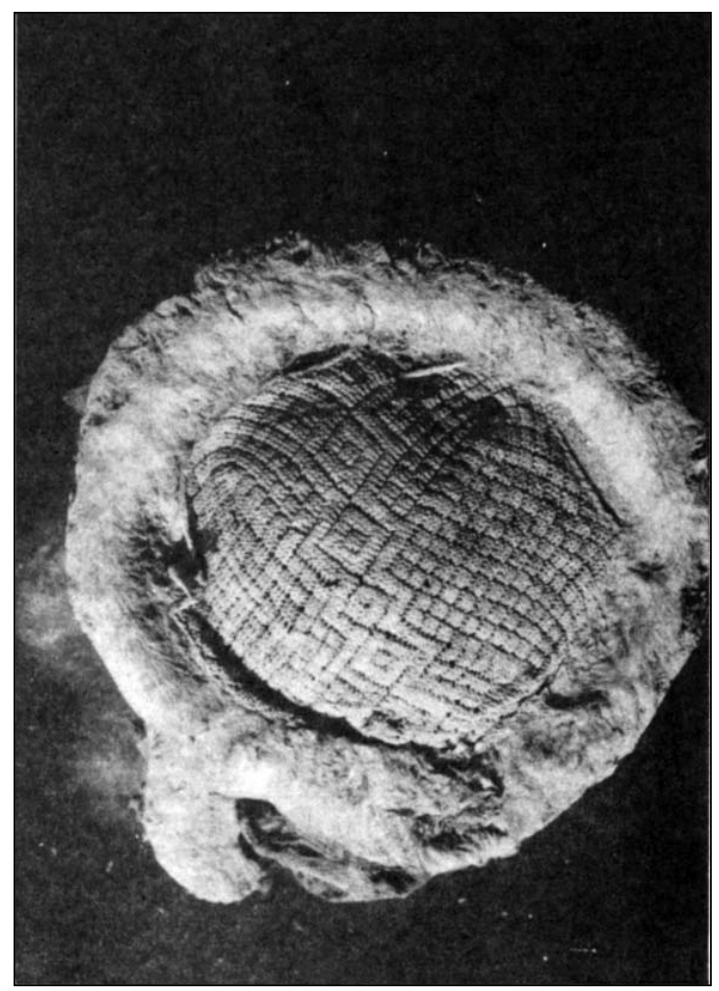

Figura 2. Un tocado asociado al estilo del Grupo B en Coyo (tumba 4107). El tocado está formado por dos trenzas, una para el "barbiquejo", y su centro está tejido en técnica de anudado (knotting). 
Los habitantes de Songo demuestran que la división del trabajo existe. Por cierto, don Luis le dijo a Allen que: "en febrero cuando no hay trabajo de agricultura, yo me vuelvo mujer y me pongo a tejer". Pero teoría y práctica se funden constantemente como recuerda Allen (1988: 78): "Cuando aprendí a tejer hace dos años, Basilia exclamó con alegría 'Bueno, por fin ya eres mujer!' Su esposo, hijo y compadre se sentaron, junto a ella, todos tejiendo."

Las distinciones sociales de las características del género de las poblaciones de los Andes pueden ser entendidas más claramente, no sólo como él o ella sino como más de él o más de ella dependiendo de la situación. Meisch (1991: 11) reconoce un tema similar en la descripción de Collins (1988: 140) de una comunidad peruana de Moho:

"Aunque la gente aymara le da importancia a los papeles complementarios de hombres y mujeres en su arte, religión y filosofía, la verdadera división del trabajo es por sexo y es bastante flexible. Uno oye comúnmente comentarios como 'los hombres aran la tierra y las mujeres siembran' y 'las mujeres tejen mejor que los hombres', sin embargo, hay algunas labores que no son ejecutadas por género o por sexo en particular, pero más bien varían según las circunstancias."

El género complementario andino entonces provee una situación donde los miembros de cada sexo mantienen un conocimiento del papel del sexo opuesto (y pueden, de hecho, llevarlos a cabo) y definitivamente llegar a un entendimiento de excelencia: en la mayoría de las comunidades indígenas del sur de los Andes de hoy en día, un buen tejedor es apreciado por todos. Es sabido que las mujeres son las tejedoras principales en términos del uso de los telares tradicionales. Ellas son responsables por la producción de las prendas de vestir de sus familias, pero los hombres también tejen sombreros y trenzan cuerdas de fuertes hebras múltiples. Miembros de ambos sexos hilan, pero hilar así como tejer, es considerado un trabajo femenino en el sur de Perú, Bolivia y en el norte de Chile.

Es posible que en Coyo las mujeres de varios grupos hayan sido las responsables de la mayoría de la producción textil, del tejido y de los bordados de diseños específicos, relacionados a categorías étnicas. La presunción de que las mujeres fueron las tejedoras en el contexto andino prehistórico también se reconoce por la distribución de la variedad de artefactos en el contexto arqueológico, como de él o ella, así como lo hacen las comunidades indígenas andinas modernas. Los entierros de los hombres en Coyo Oriental suelen tener torteras para hilar (nueve torteras en tumbas de hombres y 27 en tumbas de mujeres), pero los entierros de mujeres frecuentemente contienen fibras, agujas, madejas de hilos de colores, partes de telares, espadas y torteras de husos.

Los textiles enterrados con las "dos hermanas locales" atestiguan que estas mujeres fueron tejedoras e hiladoras extraordinarias y para enfatizar este hecho, su tumba fue equipada con cinco torteras.

\section{La mujer de la manta roja: Estilo altiplánico}

La "mujer de la manta roja" murió aproximadamente en 670 DC (Beta 33853: 1310 70 AP) y vivió aproximadamente hasta los 40 o 44 años. Algunas señales de tensión en el esqueleto indican artritis causada por la carga de materiales pesados en el torso, espalda y hombros durante toda su vida (Arriaza 1990Ms). Debe recordarse que tanto piedras para martillar y minerales de cobre se asocian con labores mineras y constituyen parte del inventario de Coyo (Oakland 1990Ms). Estas evidencias indican que esta mujer pudo haber trabajado en esta actividad junto a miembros de ambos sexos de su familia.

Posiblemente esta mujer tejió las cuatro túnicas y por lo menos dos de las tres mantas o faldas encontradas en su tumba (Figura 3). Los textiles cubrieron su cuerpo flexionado, formando un fardo funerario compacto atado con cuerdas de fibra de camélidos. Aparentemente, los hombres y las mujeres usaron ropas similares, túnicas formadas por un rectángulo sencillo con un orificio para el cuello y decoradas con listas en los dos lados (Figura 4). Las túnicas fueron rematadas doblando y cosiendo los lados, dejando espacio libre para los brazos. Los peinados de los hombres y las mujeres eran distintos y posiblemente la forma de arreglarse el cabello constituía lo más distintivo de los dos sexos. En efecto, los hombres usaban el cabello largo, trenzado en varias tiras y enrollado en una sola trenza por detrás de la cabeza. El pelo de las mujeres, como lo representa "la mujer de la manta roja", era partido por el centro y enrollado en dos chongos o abultamiento a cada lado de la cabeza. Todos los textiles de faz de urdimbre encontrados en la tumba de "la mujer de la manta roja" forman un grupo de prendas de 
vestir de colores y secuencias de listas similares. La túnica de listas simétricas con el cuello bordado con el patrón de tablero (Figura 5) identifica a esta mujer como copartícipe del mismo estilo de vestir con otras personas de su grupo (Figura 6). Además, el fardo funerario contenía dos túnicas con listado asimétrico y otra túnica sin decoración así como también dos mantas de faz de urdimbre listadas y una manta extraordinaria de tapiz rojo de estilo Tiwanaku (Figura 7). La semejanza en el diámetro de los hilados, los colores y los diseños torcidos de las prendas de vestir, sugieren la posibilidad de haber sido tejidos solamente por esta mujer. Las variaciones en los puntos de los tejidos y colores pueden ser explicados como producto de diferentes períodos de fabricación. Un manto y una túnica con decoración asimétrica encontrados parecen ser los repuestos de sus textiles más usados y remendados. La manta de tapiz rojo contrasta con las otras prendas de vestir en color y patrón, así como en técnica y es uno de los textiles más finos encontrados en las colecciones del Museo de San Pedro de Atacama.

No hay nada que sugiera que la mujer enterrada con la manta roja haya sido también tejedora de tapiz y que pudo haber creado la manta roja. El tapiz en cualquier forma es poco común en todas las colecciones de San Pedro de Atacama. La fineza y uniformidad de los hilos, la brillantez de los teñidos, la exactitud de la ejecución de los diseños representativos y la estructura del tapiz mismo sugieren que esta prenda de vestir fue traída del núcleo mismo de Tiwanaku. Una pequeña rotura reparada con un hilo rojo diferente sugiere que la mujer y la manta fueron trasladados del lugar de su

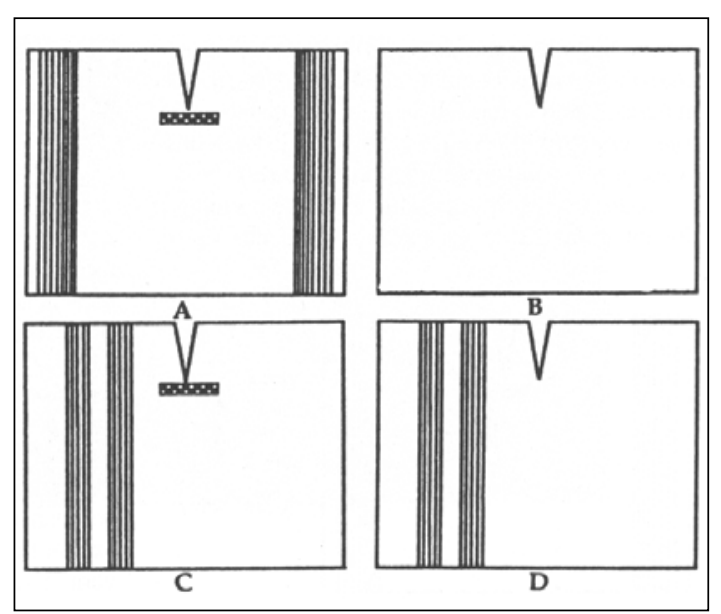

Figura 3. Dibujo de cuatro formas de túnicas encontradas en la tumba 4012 con la "mujer de la manta roja".

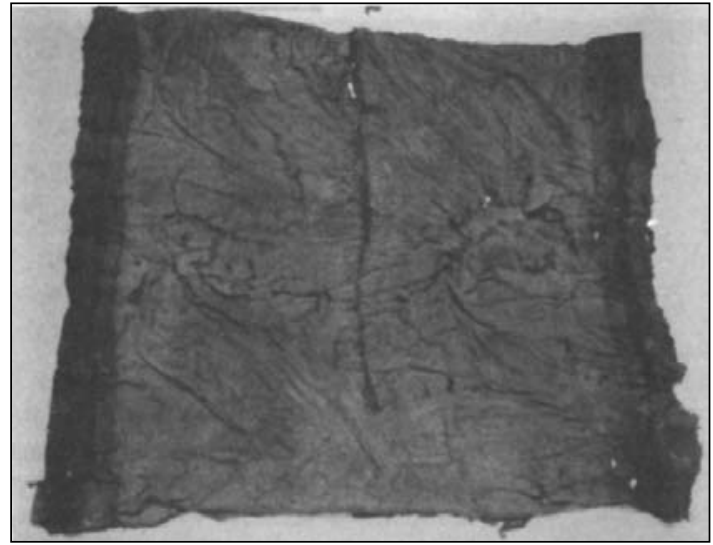

Figura 4. Una túnica de estilo del Grupo B encontrada en la tumba 4012.

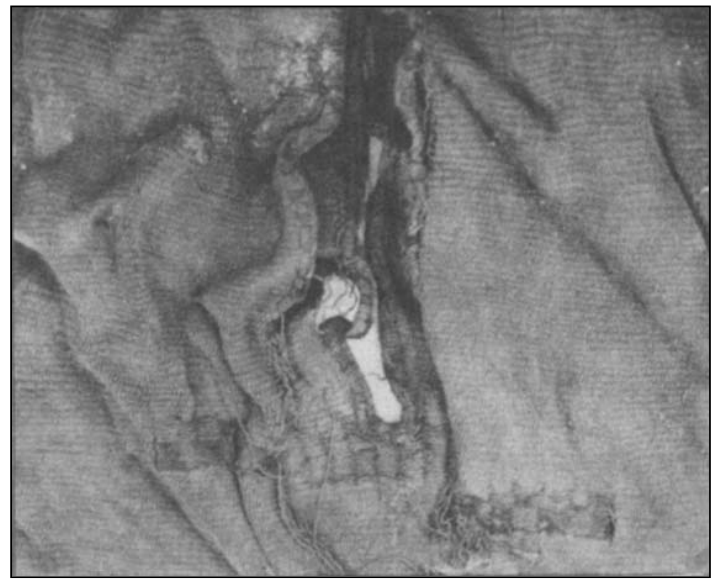

Figura 5. Patrón "tablero de ajedrez" de la túnica de la tumba 4012.

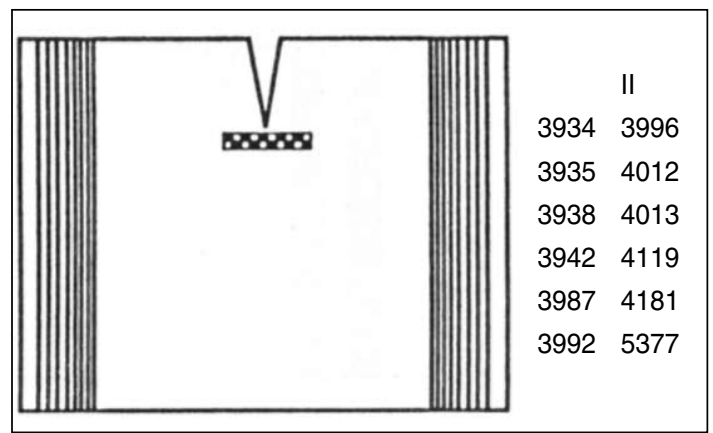

Figura 6. Diagrama de la túnica ilustrada en la Figura 4, y una lista de tumbas con túnicas similares en estilo al Grupo B.

lugar de origen, después de haber sido fabricada la pieza en cuestión. Una reconstrucción del evento podría sugerir que la manta arribó como un regalo y/o intercambio importado del altiplano o que la mujer la pudo haber traído con ella a este oasis de 
Atacama. Otros tapices de Tiwanaku descubiertos en Coyo Oriental, como la túnica de la tumba 5382 (Figuras 8 y 9) se asocian con la misma túnica de faz de urdimbre que tenía "la mujer de la manta roja" y estaba bordada con el mismo patrón de tablero en el cuello. En otras regiones aledañas se ha observado este mismo patrón, también localizado en el cuello como un rasgo común en el bordado de las túnicas de tapiz de Tiwanaku de otras colecciones recuperadas en sus áreas de influencia en el centro-sur de los Andes (p.e., túnica de Pica 8 descrita por Berenguer y Dauelsberg 1989: Figura 8a). Existen varias reconstrucciones posibles en torno a estos eventos de Coyo, pero la
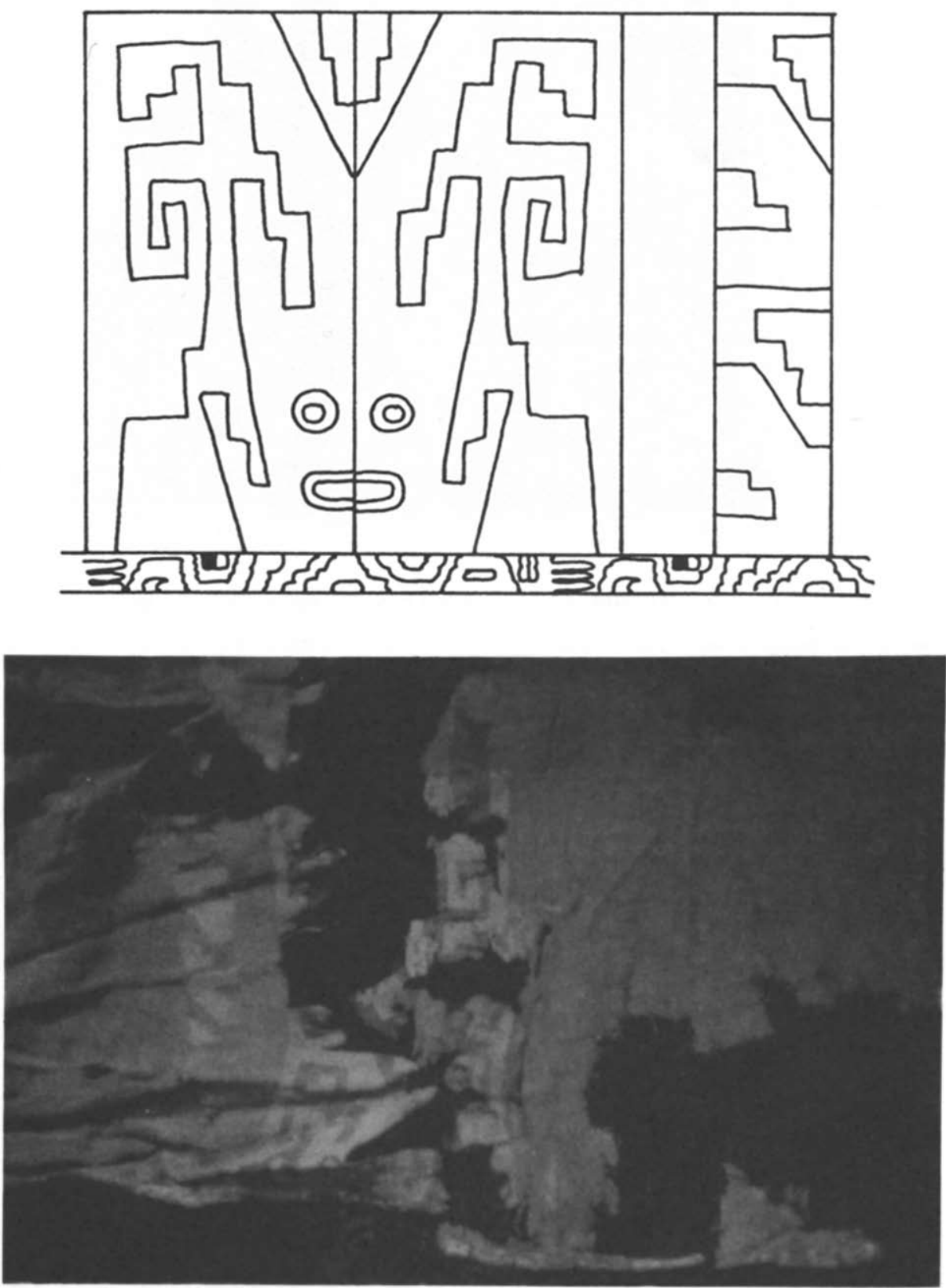

Figura 7. Dibujo de un módulo de diseño y fotografía de la manta roja de la tumba 4012. El tapiz es de colores azul, verde, rojo y amarillo; el fondo es de color rojo fuerte. 
tradición común de textiles ligando "la mujer de la manta roja" con otras de estructuras idénticas y rasgos iconográficos distintos del estilo local de San Pedro, atestiguan que la influencia de los pueblos altiplánicos y su posible ocupación en el oasis de Coyo fue muy importante. En verdad, la mujer y su grupo pudieron haber vivido por un largo tiempo en San Pedro, pero la noción de diferencia, separación, inclusividad y asociación con el lugar de origen (Tiwanaku) fue recordado y expresado a través de un estilo textil exótico al lugar y recargado de símbolos atribuidos a un "centro" lejano pero no ignorado.

\section{El estilo local y las "dos hermanas"}

San Pedro de Atacama es un conjunto de oasis del desierto conocido por sus registros culturales e ideológicos provenientes de otras regiones trasandinas (Núñez y Dillehay 1979). En este sentido ofrece un sitio de prueba ideal para la teoría que trata de explicar la diversidad cultural como un producto de interacción multiétnica. Desde el punto de vista de Weissner (1989), el sentido de identificación es creado en referencia específica a comparaciones con otros grupos. Dentro de una sociedad cerrada, muy poca diferencia es expresada por individuos familiarizados entre sí, pero en situaciones donde el intercambio y acceso abienes complementarios es constante además de la competencia por recursos, los individuos y sus grupos establecen una identidad distintiva, comúnmente basada en la elaboración de estilos textiles (Weissner 1974, 1989; Wobst 1977). Un estilo en prendas de vestir es representado varias veces dentro de los diversos trajes encontrados en las colecciones arqueológicas provenientes de los oasis de San Pedro de Atacama y probablemente representan genuinamente al estilo local de San Pedro (Figura 10). La evidencia más temprana de este estilo se encuentra en los bordados de la túnica de un hombre prominente enterrado en el cementerio Quitor 6, en el ayllu homónimo (tumba 2523), datado aproximadamente por los 340 DC (Le Paige 1963; Berenguer et al. 1988: 344). Un tipo de bordado idéntico asociado esta vez con una serie específica de túnicas faz de urdimbre se ha reconocido en diversos ayllu de San Pedro de Atacama en Coyo, Quitor 1, Quitor 3, Solcor y Catarpe. Es decir, hay evidencias de que el estilo local fue usado en el mismo tiempo en Coyo como "la mujer de la manta roja" (ver túnica de la tumba 4064 fechada en 670 DC, Oakland 1992; Figura 8). Aparentemente el estilo local se desarrolló más con un interés específico en estructuras de diseños torcidos dentro de las secuencias de listas observadas en los períodos anteriores. Se propone que "las dos hermanas" de Coyo pudieron haber sido responsables de estas innovaciones por el énfasis de traer estos diseños nuevos a los oasis atacameños. Comparaciones con la cerámica, fechas radiocarbónicas, análisis de otros estilos locales de diseño de textiles en Coyo Oriental, así como otras localidades de San Pedro, sitúan la sepultura de "las dos hermanas" entre 800-1000 DC.

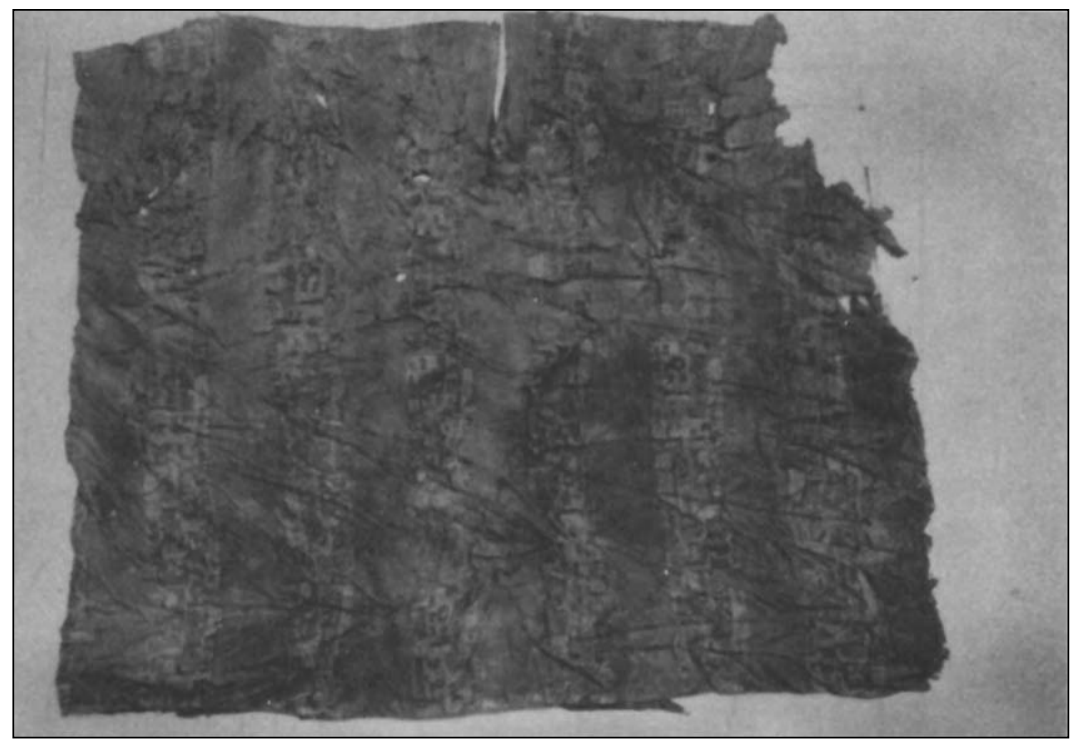

Figura 8. La túnica de Tiwanaku con seis franjas decoradas en técnica de tapicería de la tumba 5382 de Coyo Oriental. 


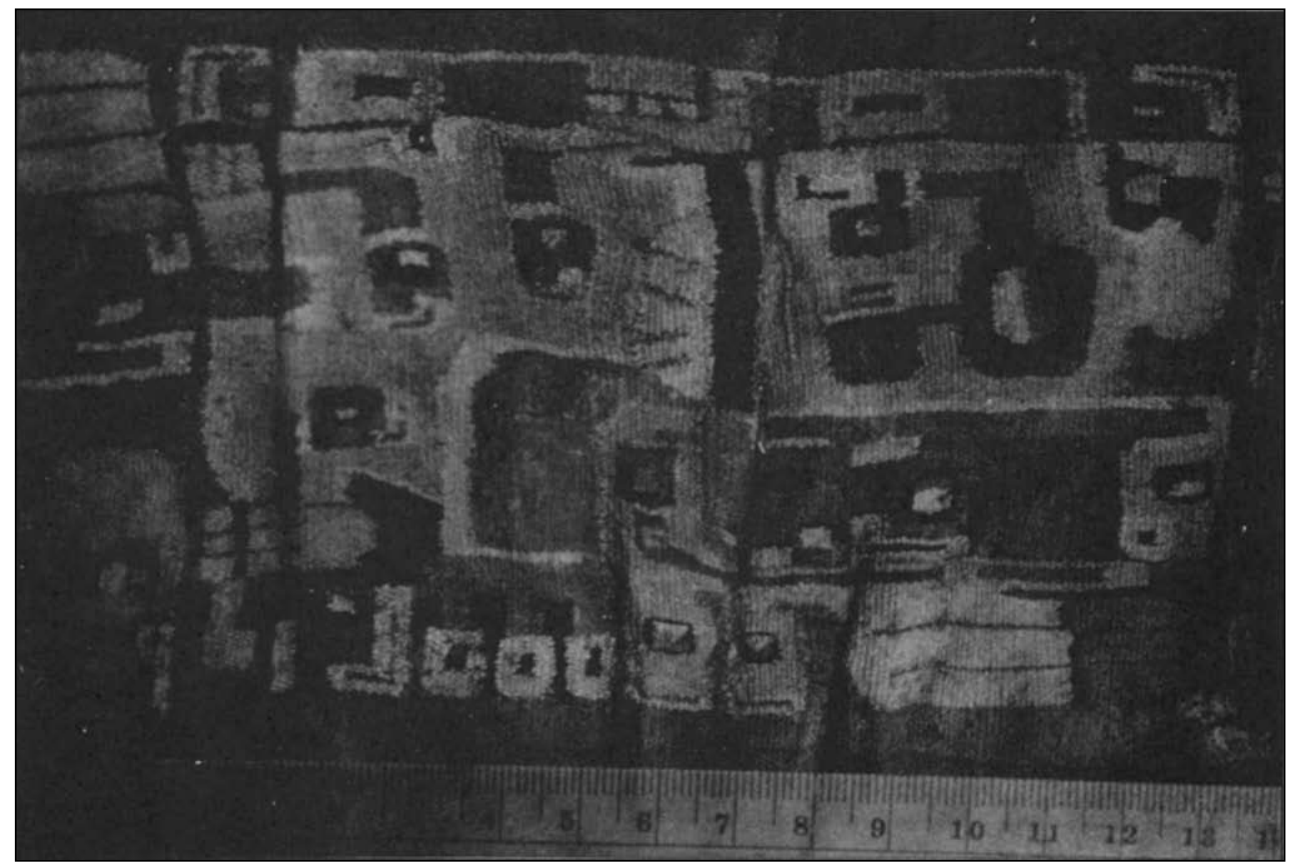

Figura 9. Detalle de la túnica ilustrada en la Figura 8.
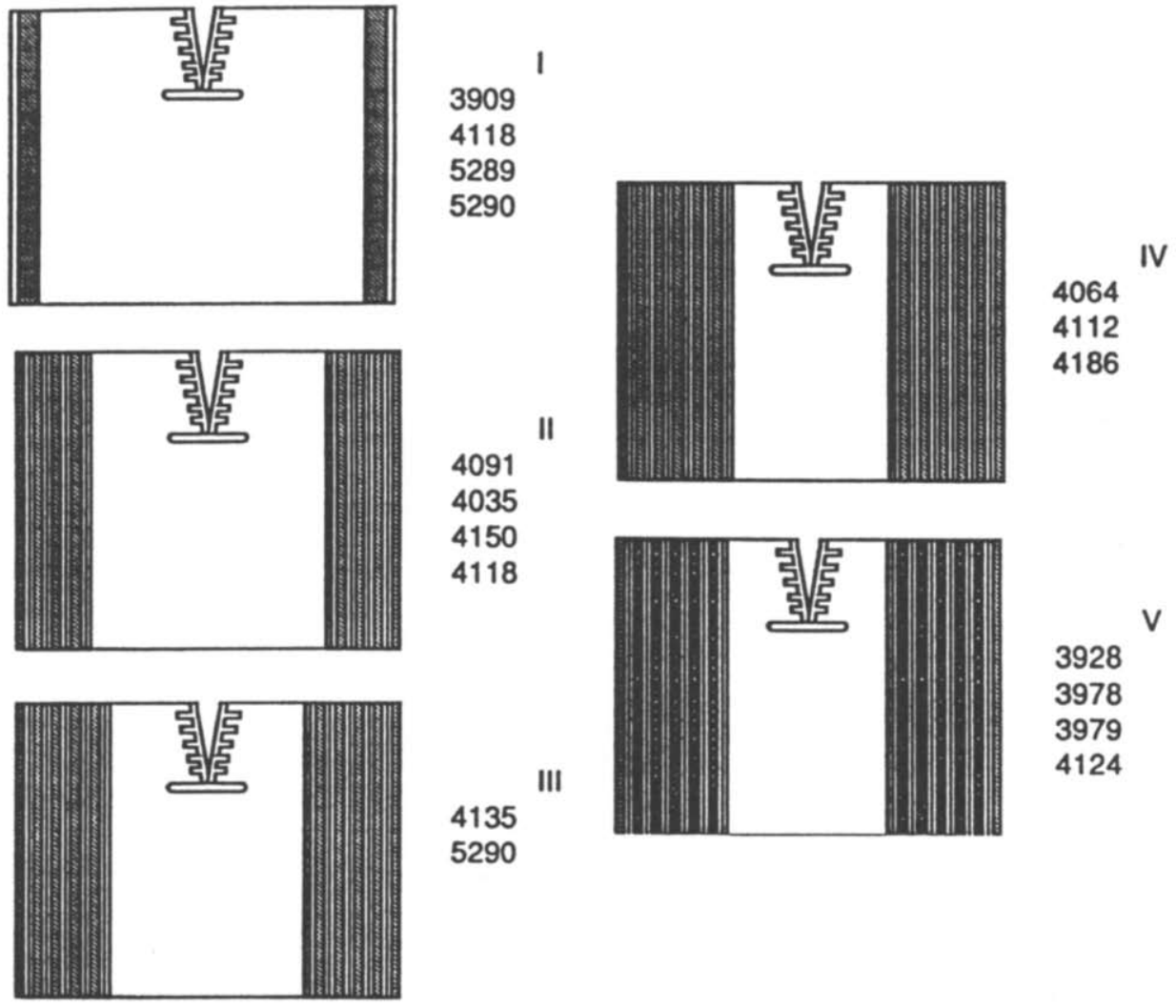

5290

Figura 10. Diagrama de túnicas de los tipos I-IV encontradas en las tumbas de Coyo Oriental (Oakland 1992). 
Podría decirse que las "dos hermanas", que murieron entre los 40 y 44 años de edad, crearon una bella variación inicialmente única dentro del estilo local, caracterizado básicamente por túnicas faz de urdimbre. En vez de tejer listas llanas en sus túnicas, con un tejido simple, y en vez de basarse en el estilo local de bordados con diseños en las uniones laterales, ellas tejieron urdimbres complementarias en las áreas comúnmente reservadas a listas de colores (Figura 11).

Las prendas de vestir son distintivas y elegantes, aún en su estado fragmentado. Puede darse el caso que una prenda sea un poco más fina que la otra y esté adornada con 10 listas verticales mientras que las otras prendas tengan sólo seis.

Otros ejemplos de diseños del estilo local en los oasis de San Pedro, que probablemente fueron contemporáneos con "las dos hermanas", incluyen escasas listas angostas con motivos creados por urdimbres complementarias y colores azul-verde pálido. Los diseños creados por urdimbres complementarias de las "dos hermanas" se integraron en listas más anchas con diseños más elaborados en anaranjado, rojo y azul. Dada la variedad de preservación dentro de los oasis de San Pedro y otros lugares del norte de Chile, es dudoso que se pueda ubicar el lugar de origen de los patrones de las "dos hermanas". Posiblemente, ellas adoptaron su propia tradición textil a los estilos del Atacama reflejando una alianza matrimonial, tal vez lo asimilaron en alguna peregrinación al núcleo Tiwanaku, en alguna feria regional, en un flujo caravanero extracircumpuneño, en alguna transacción interétnica o con colonos de una instalación estable en los oasis.

La tendencia a usar urdimbres complementarias y técnicas y diseños complejos en faz de urdimbre fue aparentemente un fenómeno que se esparció por el norte de Chile, empezando alrededor del 1000 DC (Ulloa 1982). Entonces, es posible que las túnicas de las "dos hermanas" representen las primeras innovaciones en este sentido entre los oasis atacameños.

Cualquiera de estas reconstrucciones prehistóricas es posible, o quizás ninguna, pero cada una sugiere un papel activo de cada individuo así como estas mujeres, en el traslado de bienes de estatus e identidad en un universo surandino interconectado por diversas agrupaciones étnicas.

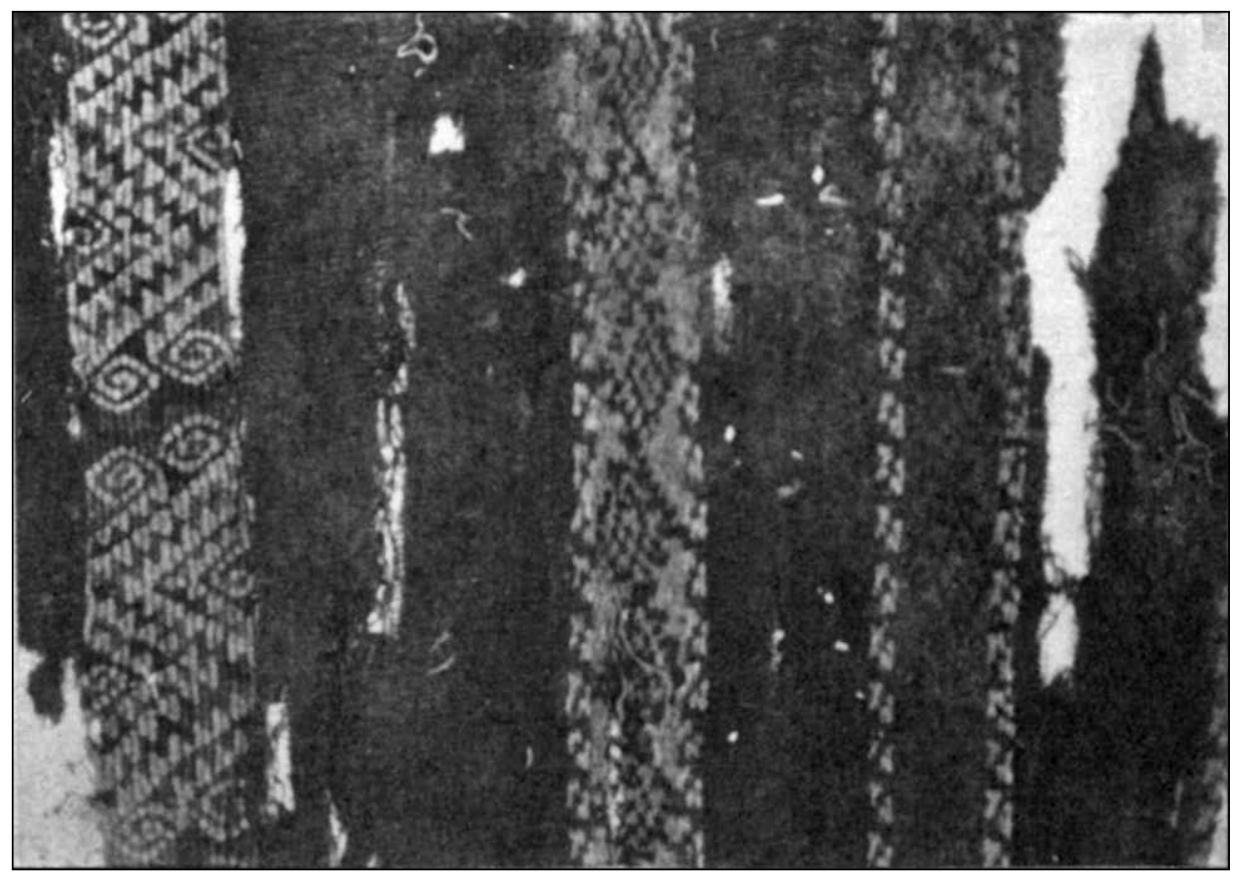

Figura 11. Detalle de la túnica de la tumba 3979 tejida por una de "las dos hermanas". 


\section{Conclusión}

El análisis presentado en este artículo se ha enfocado en los textiles de tres mujeres que vivieron y murieron en los oasis del Desierto de Atacama hace más de un milenio. El análisis es exclusivo porque fue escogido para ilustrar la presencia de individuos cuyos estilos textiles diferentes los identifican como parte de grupos más densos con cabeceras lejanas, pero que vivieron y fueron enterrados en un oasis de Atacama en donde siguieron manteniendo una tradición textil distintiva, posiblemente relacionada con sus filiaciones étnicas originarias.

Es posible que Coyo Oriental no sea el único lugar de San Pedro donde convivieron comunidades multiétnicas. Parece que muchos sitios parecidos existen en el Area Centro Sur Andina. Es decir, un análisis de los estilos textiles preservados en comunidades prehistóricas del sur de Perú, norte de Chile, Bolivia y noroeste de Argentina podría iniciar un proceso de identificación de antiguas identidades, tal como ellos originalmente se identificaron a sí mismos, permitiendo así a los antiguos pueblos andinos presentar su propia lectura de la prehistoria.

Agradecimientos Este artículo está basado en la ponencia The Women of Coyo, expuesta en 1992 en la Conferencia de la Textile Society of America, en Seattle. La investigación y la beca para la conservación de la colección textil de Coyo Oriental (Museo Arqueológico de la Universidad Católica del Norte) fue patrocinado por la National Endowment for the Humanities (RO-21963-89). El consejo y asistencia de Agustín Llagostera y M. Antonietta Costa fueron siempre apreciados. Vivian Reciné y Vicki Cassman colaboraron en la desenvoltura del fardo de la "mujer de la manta roja" y en general en la conservación de los tejidos de Coyo. Bernardo Arriaza y M. Antonietta Costa identificaron los principales rasgos antropológicos físicos de los cuerpos de Coyo Oriental. Cecilia Ramírez asistió el proceso de análisis de los artefactos, mientras que las fotos son de mi esposo Michael Rodman. A todos, y en especial a la gente de San Pedro, mis agradecimientos más sentidos.

\section{REFERENCIAS CITADAS}

ALLEN, C., 1988. The hoid life has. Smithsonian Institution Press, Washington D. C.

ARRIAZA, B., 1990Ms. Paleosalud de los habitantes de Coyo Oriental: San Pedro de Atacama. Interpretive Research Project RO 21963-89. Washington D. C.

BITTMANN. B., L. NUÑEZ y G. LE PAIGE, 1978. Cultura atacameña. Depto. de Extensión Cultural, Serie El Patrimonio Cultural Chileno, Ministerio de Educación, Santiago.

BERENGUER, J. y P. DAUELSBERG, 1989. El Norte Grande en la órbita de Tiwanaku. En Culturas de Chile. Prehistoria. Desde sus orígenes a los albores de la Conquista, J. Hidalgo, V. Schiappacasse, H. Niemeyer, C. Aldunate e I. Solimano (Eds.), pp. 129-180. Editorial Andrés Bello, Santiago.

BERENGUER, J., A. DEZA, A. ROMAN y A. LLAGOSTERA, 1986. La secuencia de Myriam Tarragó para San Pedro de Atacama: Un test por termoluminiscencia. Revista Chilena de Antropología 5: 17-54.

1988. Testing a cultural sequence for the Atacama Desert. Current Anthropology 29 (2):341-346.

BROWMAN, D., 1980. Tiwanaku expansion and altiplano economic pattern. Estudios Arqueológicos 5: 107-12.

COLLINS, J., 1988. Unseasonal migrations: The effects of rural labor scarcity in Peru. Princeton University Press, Princeton.
CONKEY, M. y C. HASTORF, 1990. Uses of style in archaeology. Cambridge University Press, Cambridge.

GERO, J. y M. CONKEY, 1991. Engendering archaerology: Women and prehistory. Basil Blackwell, Oxford.

LE PAIGE, G., 1963. La antigüedad de una tumba comprobada por $\mathrm{C}^{14}$ y el ambiente que la rodea. Revista Universitaria 26.

MEDLIN, M. A., 1991. Ethnic dress and Calcha festivals, Bolivia En Textile traditions of Mesoamerica and the Andes, $\mathrm{M}$. Blum Schevill, J. C. Berlo y E. B. Dwyer (Eds.), pp. 261279. Garland Publishing Inc., Nueva York.

MEISCH, L., 1986. Weaving styles in Tarabuco, Bolivia. En The Junius Bird Conference on Andean Textiles, A. Rowe (Ed.), pp. 243-274. The Textile Museum, Washington D. C.

1991Ms. The missing half: Pre-Hispanic and contemporary traditions of Andean males as spinners and weavers. Ponencia presentada en el International Congress of Americanists (1991). Nueva Orleans.

MILLER, V., 1988. The role of gender in precolombian art and architecture. University Press of America, Maryland.

NUÑEZ, L., 1992. Cultura y conflicto en los oasis de San Pedro de Atacama. Editorial Universitaria, Santiago.

NUÑEZ, L. y T. DILLEHAY, 1979. Movilidad giratoria, armonía social y desarrollo en los Andes Meridionales: Patrones de 
tráfico e interacción económica (ensayo). Universidad del Norte, Antofagasta.

OAKLAND, A., 1986. Tiwanaku tapestry tunics and mantles from San Pedro de Atacama, Chile. En The Junius Bird Conference on Andean Textiles, A. Rowe (Ed.), pp. 101-122. The Textile Museum, Washington D. C.

1990Ms. Tiwanaku Textiles, a view from the provinces. Ponencia presentada al $30^{\text {th }}$ Annual Meeting of the Institute of Andean Studies. Berkeley.

1992. Textiles and ethnicity: Tiwanaku in San Pedro de Atacama, north Chile. Latin American Antiquity: 3 (4): 316340 .

SACKETT, J., 1990. Style and ethnicity in archaeology: The case for isochrestism. En The uses fo style in archaeology, M. Conkey y C. Hastorf (Eds.), pp. 33-50. Cambridge University Press, Cambridge.

SEIBOLD, K., 1992. Textiles and cosmology in Choquecancha, Cusco, Perú. En Andean cosmologies through time, R. Dover, K. Seibold y J. McDowell (Eds.), pp. 166-201. Indiana University Press, Bloomington.
TARRAGO, M., 1968. Secuencias culturales de la etapa agroalfarera de San Pedro de Atacama, Chile. Actas y Memorias del XXXVII Congreso Internacional Americanistas, vol. 2. Buenos Aires.

ULLOA, L., 1982. Estilos decorativos y formas textiles de poblaciones agromarítimas en el extremo norte de Chile. Chungara 8: 109-136.

WIESSNER, P., 1984. Reconsidering the behavioral basis for style: A case study among the Kalahari San. Journal of Anthropological Archaeology 3: 190-234.

1989. Style and changing relations between the individual and society. En The meanings of things, I. Hodder (Ed.). Unwin Hyman Inc., Londres.

WOBST, M., 1977. Stylistic behavior and information exchange. Anthropological Papers 6: 317-342.

ZORN, E., 1990. Modern traditions: The impact of thetrade in traditional textiles on the Sakaka of Northern Potosí, Bolivia. En Proceedings of the Textile Society of America Biennial Symposium (1990), pp. 241-252. Washington D. C. 